Proceedings

\title{
Effect of Gamma Sterilization on CBD-Loaded PLGA Microparticles ${ }^{+}$
}

\author{
Ana Isabel Fraguas-Sánchez, Ana Fernández-Carballido and Ana Isabel Torres-Suárez *
}

Citation: Fraguas-Sánchez, A.I.; Fernández-Carballido, A.; Torres

Suárez, A.I. Effect of Gamma Sterilization on CBD-Loaded PLGA

Microparticles. Proceedings 2021, 78, 31. https://doi.org/10.3390/ IECP2020-08801

Published: 1 December 2020

Publisher's Note: MDPI stays neutral with regard to jurisdictional claims in published maps and institutional affiliations.

Copyright: $\odot 2020$ by the authors. Licensee MDPI, Basel, Switzerland. This article is an open access article distributed under the terms and conditions of the Creative Commons Attribution (CC BY) license (http://creativecommons.org/licenses/by/4.0/).

\author{
Department of Pharmaceutics and Food Technology, Complutense University, 28040 Madrid, Spain \\ aifraguas@ucm.es (A.I.F.-S.); afernand@ucm.es (A.F.-C.) \\ * Correspondence: galaaaa@ucm.es \\ † Presented at the 1st International Electronic Conference on Pharmaceutics, 1-15 December 2020; \\ Available online: https://iecp2020.sciforum.net/.
}

\begin{abstract}
Cannabidiol (CBD) has emerged as a potential therapeutic agent. However, its low aqueous solubility hinders the development of effective parenteral formulations. The use of polymeric microparticles as CBD carriers could resolve this challenge and allows to obtain an extended CBD release after a single administration. Poly(lactic-co-glycolic acid) (PLGA), FDA approved for various medical applications, is one the most used. Ionizing radiation is as an effective sterilizing method for PLGA microparticles, which is essential for their parenteral administration. The aim of this work was to evaluate the effect of gamma sterilization on the characteristics of CBD-loaded microparticles. No differences in particle morphology and particle size were detected between sterile and nonsterile formulations. All microparticles exhibited a spherical shape, a smooth surface, and an average particle size around $25 \mu \mathrm{m}$. DSC analysis showed the absence of the CBD melting peak in sterile and non-sterile CBD-microparticles. However, a reduction on PLGA glass transition was appreciated in sterile formulations. A significant lower CBD content was also detected in sterile microparticles, indicating a CBD degradation during sterilization. Finally, a faster CBD release was appreciated in sterile microparticles compared with their counterparts, due to the faster PLGA degradation in sterilized microparticles. Due to the CBD degradation during sterilization process and the acceleration of the release of this drug from PLGA microparticles, gamma irradiation is not an adequate method to sterilize CBD-PLGA-microparticles.
\end{abstract}

Keywords: cannabinoids; drug delivery; polymeric microparticles; sterilization

\section{Introduction}

In the last decades, cannabinoids have emerged as potential therapeutic agents [1]. Among all cannabinoids, CBD, is one of the promising compounds, due to its lack of psychotropic effect and its analgesic, neuroprotective, anticonvulsant, anxiolytic and anticancer properties; being useful for the treatment of epilepsy, neuropathic pain, anxiety disorders and cancer among others [2,3].

Despite CBD potential clinical interest, its low aqueous solubility and stability problems make difficult the development of effective parenteral formulations [4]. In fact, the administration of CBD to animals requires the use of organic solvents like ethanol and solubilizing agents like Cremophor [5]. In this way, the development of polymeric microparticles as CBD carriers may resolve these challenges, eliminating the use of these solubilizing agents. These systems also allow an extended CBD release after a single administration [6,7], which is especially interesting in long term treatments.

Among all the available polymers, poly(lactic-co-glycolic acid) (PLGA), approved by FDA and EMA for various medical applications, is one the most used for drug delivery due to its high biocompatibility and tunable structural characteristics [8]. In fact, PLGA microparticles have showed to be an excellent carrier for the vehiculi zation of CBD [6]. 
Drug delivery systems intended for parenteral administration required to be adequately sterilized. Among all available sterilization techniques, gamma irradiation has been proposed as an effective sterilizing method for PLGA microparticles [9].

Therefore, the aim of this work was to evaluate the effect of gamma sterilization on the characteristics of CBD-loaded-PLGA-microparticles.

\section{Materials and Methods}

\subsection{Materials}

Poly-(lactide-co-glycolic acid-resomer) RG ${ }^{\circledR}-502$ (PLGA-502) were obtained from Evonik $^{\circledast}$ Industries (Essen, Germany), CBD was purchased from THC-Pharma (Frankfurt, Germany) and Polyvinyl alcohol (PVA, Mw $=30,000-70,000$ ) was supplied by Sigma-Aldrich (St. Louis, MO, USA). All organic solvents, methanol, acetonitrile, and dichloromethane (DCM) were obtained from Fisher Scientific (Frederick, MA, USA). Potassium di-hydrogen phosphate $\left(\mathrm{KH}_{2} \mathrm{PO}_{4}\right)$, disodium hydrogen phosphate dehydrate $\left(\mathrm{Na}_{2} \mathrm{HPO}_{4} \cdot 2 \mathrm{H}_{2} \mathrm{O}\right)$ and Tween ${ }^{\circledR} 80$ were provided by Panreac (Barcelona, Spain).

\subsection{Elaboration of CBD-Loaded-PLGA Microparticles}

Microparticles were prepared by the oil-in-water $(\mathrm{O} / \mathrm{W})$ emulsion-solvent evaporation technique using PLGA-RG ${ }^{\circledR}-502$ as a polymer and a CBD:PLGA ratio of 10:100 (10Mps) and 20:100 (20-Mps) [10]. PLGA (500 mg) and CBD (50 or $100 \mathrm{mg}$ ) were dissolved in $5 \mathrm{~mL}$ of DCM and the resulting organic phase was dropped onto $250 \mathrm{~mL}$ of an aqueous solution of PVA at $0.5 \%$ under stirring at 4000 and $6000 \mathrm{rpm}$ for $10-\mathrm{Mps}$ and $20-\mathrm{Mps}$, respectively. Then, the resulting O/W emulsion was stirred at $200 \mathrm{rpm}$ for $3-4 \mathrm{~h}$ and filtered using a $5 \mu \mathrm{m}$ PTFE membrane. The collected microparticles were washed thrice with 50 $\mathrm{mL}$ of demineralized to remove residual PVA and lyophilized at $-50^{\circ} \mathrm{C}$ and $0.2 \mathrm{mbar}$ with a Lyo Quest freeze-drier (Azbil Telstar, S.L., Terrasa, Spain).

\subsection{Sterilization of CBD-Loaded-PLGA-Microparticles}

Both, $10 \mathrm{Mps}$ and $20 \mathrm{Mps}$ CBD-loaded microparticles were sterilized by gamma irradiation at the recommended dose of $25 \mathrm{kGy}$ [11]. During sterilization, formulations were placed in a container with dry ice to avoid formulation heating.

\subsection{Characterization of CBD-Loaded-PLGA-Microparticles}

Both sterile and non-sterile 10-Mps and 20-Mps were characterized as follows.

\subsubsection{Particle Size and Morphology}

The particle size was evaluated by laser diffraction with a Microtrac ${ }^{\circledR}$ S3500 Series Particle Size Analyzer (Montgomeryville, PA, USA), expressing the mean particle size as a volume diameter. Span values were also calculated using the following equation:

$$
\mathrm{SPAN}=\mathrm{D} 90-\mathrm{D} 10 / \mathrm{D} 50
$$

where D10, D50 and D90 are the mean diameters determined at the $10^{\text {th }}, 50^{\text {th }}$ and $90^{\text {th }}$ particle distribution percentiles, respectively.

The shape and surface of the microparticles were evaluated by scanning electron microscopy ((SEM Jeol, JSM-6400, Tokyo, Japan) using the protocol described in [6].

\subsubsection{DSC Analysis}

Sterile and non-sterile CBD-loaded microparticles, pure CBD, raw PLGA and CBD:PLGA physical mixtures were analyzed by DSC. Briefly, around $3 \mathrm{mg}$ of each sample were weighed and placed into sealed aluminum pans. Then, they were heated from 20 to $100{ }^{\circ} \mathrm{C}$, cooled to $20^{\circ} \mathrm{C}$ and heated again up to $100{ }^{\circ} \mathrm{C}$ using a heating rate of $10{ }^{\circ} \mathrm{C} / \mathrm{min}$ and an inert nitrogen atmosphere of $70 \mathrm{~mL} / \mathrm{min}$. The polymer glass transition temperature of each sample was calculated in the second heating cycle. 


\subsubsection{Drug Content and In Vitro Drug Release}

The CBD content of non-sterile and sterile 10-Mps and 20-Mps microparticles were analyzed by HPLC using the conditions described in [6]. Briefly, $10 \mathrm{mg}$ of the formulations were weighted and dissolved in $10 \mathrm{ml}$ of DCM. Then, PLGA was precipitated by the addition of a mixture of methanol:acetonitrile:water at a ratio of 52:30:18. Then, the samples were filtered and analyzed by HPLC.

In vitro $C B D$ release was evaluated by determining the remaining $C B D$ in the microparticles. Briefly, $10 \mathrm{mg}$ of microparticles were suspended in $5 \mathrm{~mL}$ of PBS (pH.7.4) containing Tween 80 at $0.5 \%(\mathrm{w} / \mathrm{v})$ in a thermostatic bath at $37 \pm 0.5^{\circ} \mathrm{C}$ and an agitation of 100 $\mathrm{rpm}$. At predetermined time points ( $2 \mathrm{~h}, 8 \mathrm{~h}, 1,2,4,10,14,21,28$ and 31 days), the samples were centrifuged, the supernatant was removed, and CBD contents were determined as aforementioned.

Polymer degradation during the release studies was also studied. At specific time points, the microparticles were centrifuged and washed thrice with distilled water. Then, they were lyophilized and evaluated by SEM.

\subsubsection{Statistical Analysis}

Origin 2017 software (Origin Lab, MA, USA) was used to compile all the graphs and perform the statistical analyses. The data were expressed as a mean \pm S.D. (standard deviation) of three experiments $(n=3)$ and T-student test was used to evaluate significant differences $(p<0.05)$ between two groups.

\section{Results}

\subsection{Effect on the Physicochemical Properties of CBD-Loaded Microparticles}

As displayed in Figure 1, gamma irradiation did not induce changes on particle shape and surface. Both the sterile and non-sterile formulations were spheres with a smooth surface. A significant change ( $p$ value $<0.05$ ) in particle size was not detected either, showing a mean particle size for all formulations, expressed as the volume diameter, around $25 \mu \mathrm{M}$ (Table 1). Span values around or above 2 were observed, indicating that size the distribution was not monodisperse, especially in microparticles loaded with CBD at a ratio of 20:100, that exhibited a higher polydispersion.
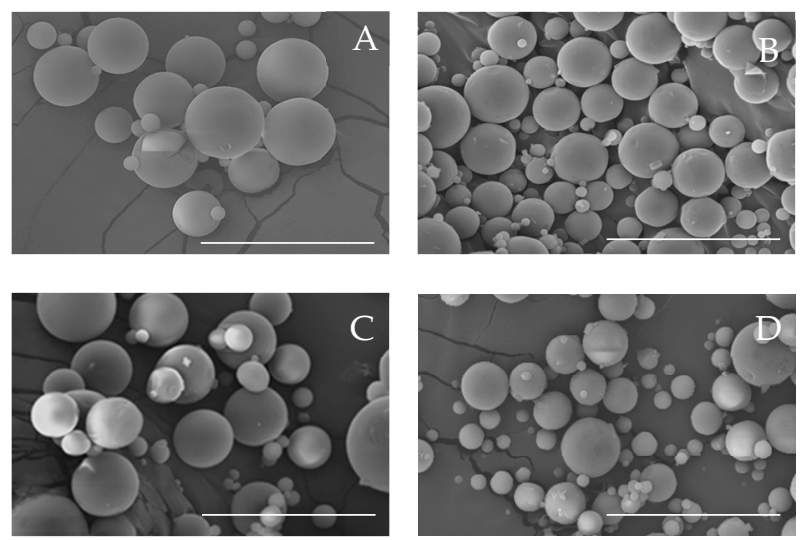

Figure 1. SEM images of 10-Mps (A); 20-Mps (B); Sterile-10-Mps (C); and Sterile-20-Mps (D). Scale bar: $50 \mu \mathrm{m}$.

In all microparticle formulations, the characteristic melting point of $\mathrm{CBD}$, detected in pure compound at $69.68^{\circ} \mathrm{C}$ and CBD: PLGA physical mixtures (data not shown), was not observed. Regarding PLGA glass transition temperature $\left(\mathrm{T}_{\mathrm{g}}\right)$, a significant decrease $(p$ value $<0.05$ ) was appreciated in CBD-loaded formulations compared with raw PLGA and unloaded formulations, indicating that CBD makes the polymer be plastic. However, a 
significant change in $\mathrm{T}_{\mathrm{g}}$ value was not appreciated in gamma-irradiated microparticles (Table 1).

Table 1. Characteristics of non-sterile and sterile cannabidiol (CBD)-loaded microparticles.

\begin{tabular}{cccccc}
\hline Formulation & $\begin{array}{c}\text { Process } \\
\text { Yield (\%) }\end{array}$ & $\begin{array}{c}\text { Particle Size } \\
(\mathbf{n m})\end{array}$ & Span & Tg $\left({ }^{\circ} \mathbf{C}\right)$ & $\begin{array}{c}\text { CBD Content (mg } \\
\text { CBD/100 mg Mps) }\end{array}$ \\
\hline Non-sterile 10-Mps & $89.25 \pm 1.01$ & 24.17 & 2.02 & 37.45 & $8.60 \pm 0.42$ \\
Sterile 10-Mps & & 23.42 & 1.95 & 37.28 & $7.42 \pm 0.13$ \\
Non-sterile 20-Mps & $81.05 \pm 5.33$ & 25.14 & 2.36 & 34.79 & $14.97 \pm 0.20$ \\
Sterile-Mps & & 24.31 & 2.42 & 35.08 & $13.43 \pm 0.43$ \\
\hline
\end{tabular}

\subsection{Effect on the Drug Content and Drug Release}

Regarding CBD content, a significant decrease was observed in both sterile 10-Mps and 20-Mps compared with their non-sterile counterparts (Table 1), indicating a CBD degradation during gamma irradiation. A $13.75 \%$ and $10.28 \%$ of CBD loss was detected in sterile formulations prepared with a CBD: PLGA ratio of 10:100 and 20:100, respectively.

A change in drug release profile was also detected in gamma irradiated formulations (Figure 2), showing that these microparticles have a faster CBD release. While in nonsterile 10-Mps formulations, an extended CBD release was observed for 40 days (with more than $80 \%$ of the drug released), their sterile counterpart exhibited almost $90 \%$ of the CBD released in 15 days. Similar data were obtained in 20-Mps formulations. While nonsterile 20-Mps microparticles also released more than $80 \%$ of the CBD in 35 days, sterile $20-\mathrm{Mps}$ almost reached $90 \%$ of the CBD release in 21 days.

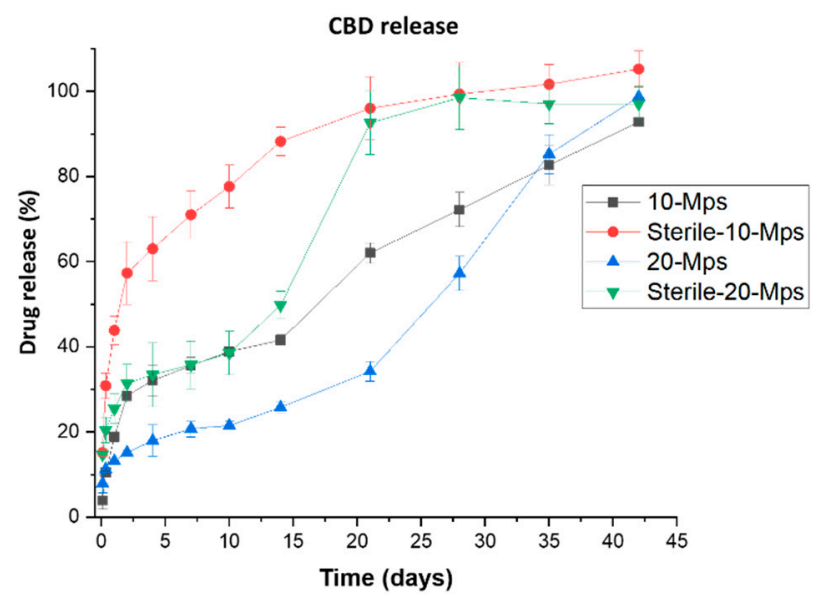

Figure 2. CBD release studies of non-sterile and sterile 10-Mps and 20-Mps.

Finally, as displayed in Figure 3, polymer degradation analyses during release studies demonstrated a higher polymer erosion in both sterile 10-Mps and 20-Mps formulations. While in sterile formulations microparticles started to lose their shape and showed a high pore formation at day 14 , non-sterile formulations, although exhibiting signs of corrugations, maintained their spherical shape and did not exhibit a high pore formation, indicating a slower polymer erosion. 

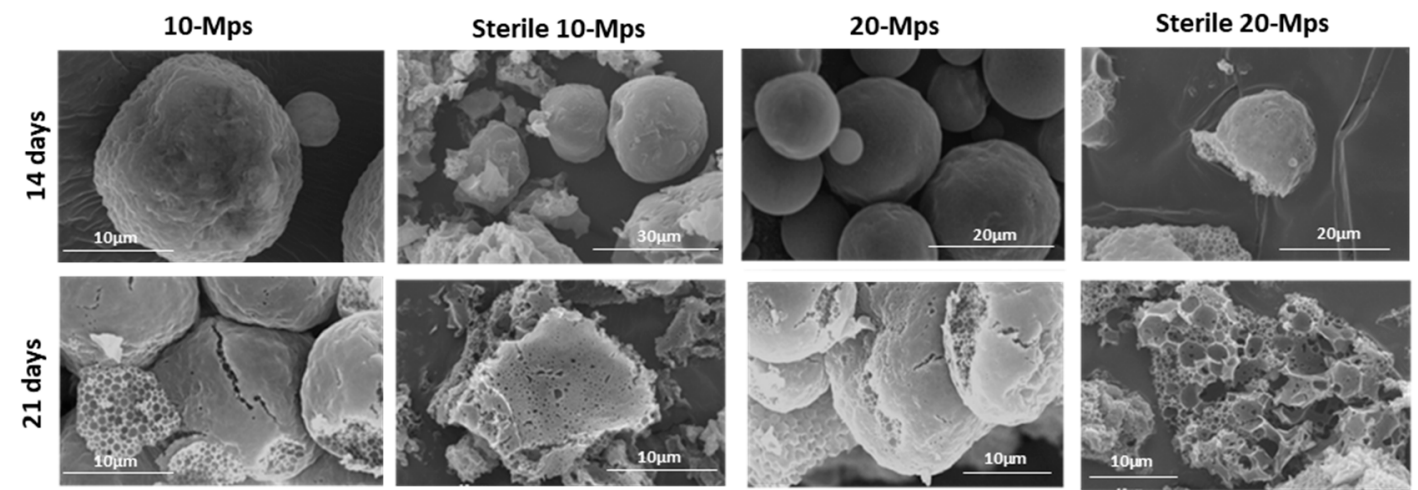

Figure 3. SEM images of non-sterile and sterile CBD-loaded PLGA microparticles during release studies after 14 and 21 days.

\section{Discussion}

Gamma irradiation is one of the sterilization methods recommended to adequately sterilize PLGA microparticles [9]. However, this process may alter physicochemical properties of the formulations, and consequently, their safe and efficacy, as it has been reported by many authors. For this reason, it is important to evaluate the effect of this sterilization technique.

Several authors have reported that gamma irradiation induces changes in the morphology of PLGA microparticles and also favors particle aggregation, which increases the mean particle size and polidispersion. These changes have been detected, for example, in naproxen, diclofenac and clonazepam-loaded microparticles which were gamma irradiated at $25 \mathrm{kgy}[12,13]$. However, in developed CBD-loaded microparticles, the use of gamma irradiation at this dose did not produce changes either in its morphology or in particle size and particle distribution, preserving gamma-irradiated formulations and the spherical shape and the particle size around $25 \mu \mathrm{m}$ of the non-sterile counterparts. Changes in particle size and polydispersity were not detected either in gamma-irradiated methotrexate PLGA microparticles [11].

DSC analyses demonstrated the absence of a CBD characteristic melting point in both non-sterile and sterile CBD-loaded microparticles, indicating that the drug was dissolved or molecularly dispersed within the polymer matrix [14] and that no crystallization occurred during sterilization. No effect on polymer glass transition temperature was also observed, probably due to the use of dry ice to protect the samples, as reported in indomethacin loaded PLGA microparticles, where non-protected samples showed a decrease in the $\mathrm{Tg}$ value.

However, gamma irradiation significantly influenced the CBD content and CBD release. On the one hand, a CBD loss of around $10 \%$ and $13 \%$ was appreciated in sterile formulations loaded with CBD at drug:polymer ratios of 20:100 and 10:100, respectively. This could be attributed to the degradation of the superficial CBD that was not completely entrapped in the polymer matrix induced by gamma irradiation. On the other hand, a faster CBD release was appreciated in sterilized formulations compared with their counterparts, which is probably attributed to a decrease in polymer molecular weight that has been reported by other authors in other gamma-irradiated formulations $[15,16]$. The lower molecular weight in sterilized formulations can also explain the faster polymer erosion that was detected in sterilized CBD-loaded microparticles.

\section{Conclusions}

Due to the $\mathrm{CBD}$ degradation during sterilization process and the acceleration of the release of this drug from PLGA microparticles, gamma irradiation is not an adequate method to sterilize CBD-PLGA-microparticles. The sterility of the microparticles could be 
achieved by the previous sterilization of the aqueous and the organic phases followed by the microparticle elaboration in aseptic conditions.

Acknowledgments: A.I. Fraguas-Sánchez has been granted with a research fellowship (Ref: FPU 14/06441), a mobility grant (EST 17/ 00553) from the Ministry of Education of Spain, and the Juan Abelló I doctoral prize from the Doctoral Academy of Spain.

Funding: This research was supported by Complutense Research Fund (Ref. FEI16/83) and by the Complutense University UCM research group "Formulation and bioavailability of new drug products" (Ref. UCM-910939). A.I. Fraguas-Sánchez has been granted with a research fellowship (Ref: FPU 14/06441).

Institutional Review Board Statement: Not applicable.

Informed Consent Statement: Not applicable.

Conflicts of Interest: The authors declared no conflicts of interest.

\section{References}

1. Fraguas-Sánchez, A.I.; Torres-Suárez, A.I. Medical Use of Cannabinoids. Drugs 2018, 78, 1665-1703.

2. Campos, A.C.; Fogaça, M.V.; Sonego, A.B.; Guimarães, F.S. Cannabidiol, neuroprotection and neuropsychiatric disorders. Pharm. Res. 2016, 112, 119-127.

3. Pisanti, S.; Malfitano, A.M.; Ciaglia, E.; Lamberti, A.; Ranieri, R.; Cuomo, G.; Abate, M.; Faggiana, G.; Proto, M.C.; Fiore, D.; et al. Cannabidiol: State of the art and new challenges for therapeutic applications. Pharmacol. Ther. 2017, 175, 133-150.

4. Fraguas-Sánchez, A.; Fernández-Carballido, A.; Martin-Sabroso, C.; Torres-Suárez, A.; Sofware, C.M.-S. Stability characteristics of cannabidiol for the design of pharmacological, biochemical and pharmaceutical studies. J. Chromatogr. B Anal. Technol. Biomed. Life Sci. 2020, 1150, 122188.

5. Viudez-Martínez, A.; García-Gutiérrez, M.S.; Fraguas-Sánchez, A.I.; Torres-Suárez, A.I.; Manzanares, J. Effects of cannabidiol plus naltrexone on motivation and ethanol consumption. Br. J. Pharm. 2018, 175, 3369-3378.

6. Fraguas-Sánchez, A.; Fernández-Carballido, A.; Simancas-Herbada, R.; Martin-Sabroso, C.; Torres-Suárez, A. CBD loaded microparticles as a potential formulation to improve paclitaxel and doxorubicin-based chemotherapy in breast cancer. Int. J. Pharm. 2020, 574, 118916 .

7. De La Ossa, D.H.P.; Lorente, M.; Gil-Alegre, M.E.; Torres, S.; García-Taboada, E.; Aberturas, M.D.R.; Molpeceres, J.; Velasco, G.; Torres-Suárez, A.I. Local delivery of cannabinoid-loaded microparticles inhibits tumor growth in a murine xenograft model of glioblastoma multiforme. PLoS ONE 2013, 8, e54795.

8. Schwendeman, S.P.; Shah, R.B.; Bailey, B.A.; Schwendeman, A.S. Injectable controlled release depots for large molecules. J. Control. Release 2014, 190, 240-253.

9. Keles, H.; Naylor, A.; Clegg, F.; Sammon, C. Studying the release of hGH from gamma-irradiated PLGA microparticles using ATR-FTIR imaging. Vib. Spectrosc. 2014, 71, 76-84.

10. Fraguas-Sánchez, A.; Fernández-Carballido, A.; Delie, F.; Cohen, M.; Martin-Sabroso, C.; Mezzanzanica, D.; Figini, M.; Satta, A.; Torres-Suárez, A. Enhancing ovarian cancer conventional chemotherapy through the combination with cannabidiol loaded microparticles. Eur. J. Pharm. Biopharm. 2020, 154, 246-258.

11. De Oliveira, A.R.; Mesquita, P.C.; Machado, P.R.; Farias, K.J.; de Almeida, Y.M.; Fernandes-Pedrosa, M.F.; Cornelio, A.M.; Socrates, T.; da Silva-Junior, A.A. Monitoring structural features, biocompatibility and biological efficacy of gamma-irradiated methotrexate-loaded spray-dried microparticles. Mater. Sci. Eng. C 2017, 80, 438-448.

12. Montanari, L.; Cilurzo, F.; Valvo, L.; Faucitano, A.; Buttafava, A.; Groppo, A.; Genta, I.; Conti, B. Gamma irradiation effects on stability of poly(lactide-co-glycolide) microspheres containing clonazepam. J. Control. Release 2001, 75, 317-330.

13. Çalış, S.; Bozdağ, S.; Kaş, H.; Tunçay, M.; Hıncal, A. Influence of irradiation sterilization on poly(lactide-co-glycolide) microspheres containing anti-inflammatory drugs. IL Farm. 2002, 57, 55-62.

14. Wang, H.; Zhang, G.; Sui, H.; Liu, Y.; Park, K.; Wang, W. Comparative studies on the properties of glycyrrhetinic acid-loaded PLGA microparticles prepared by emulsion and template methods. Int. J. Pharm. 2015, 496, 723-731.

15. Bartolotta, A.; D’Oca, M.; Campisi, M.; De Caro, V.; Giandalia, G.; Giannola, L.I.; Brai, M.; Calderaro, E. Effects of gammairradiation on trehalose-hydroxyethylcellulose microspheres loaded with vancomycin. Eur. J. Pharm. Biopharm. 2005, 59, 139146.

16. Jain, S.; Malyala, P.; Pallaoro, M.; Giuliani, M.; Petersen, H.; O'Hagan, D.T.; Singh, M. A two-stage strategy for sterilization of poly(lactide-co-glycolide) particles by $\gamma$-irradiation does not impair their potency for vaccine delivery. J. Pharm. Sci. 2011, 100, 646-654. 